\section{Ceco-colic intussusception with subsequent bowel infarction as a rare complication of colonoscopic polypectomy}

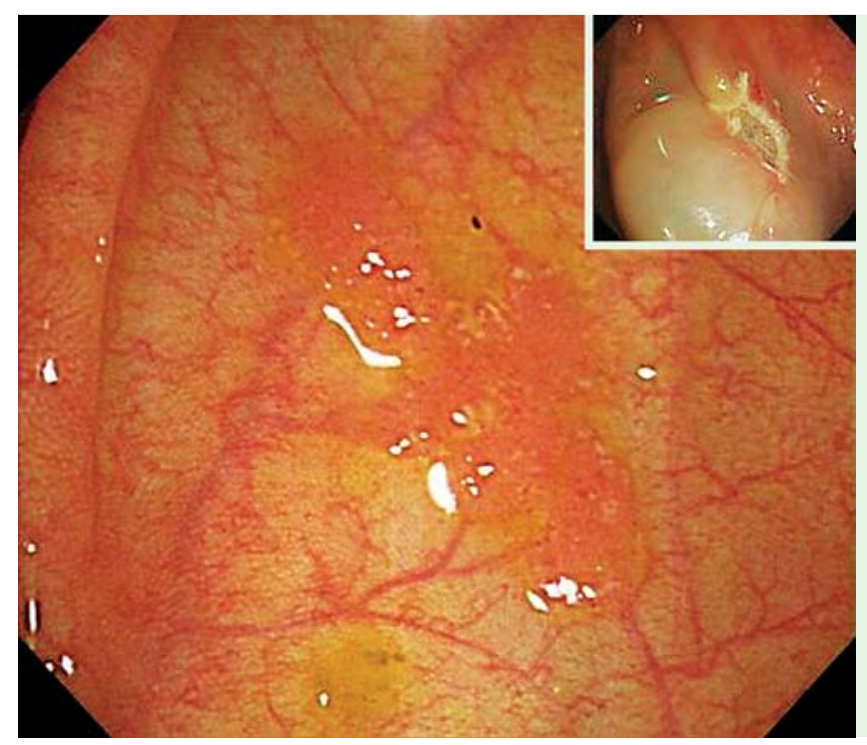
ulcer after saline-assist-
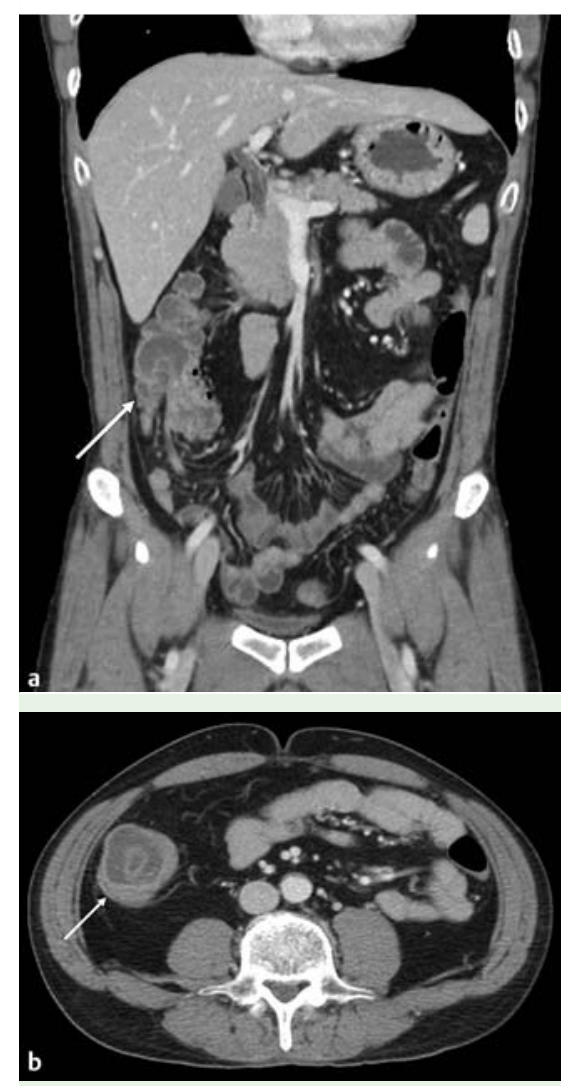

Fig. 2 Urgent abdominal computed tomography (CT) showing: a telescoping of the cecum into the ascending colon; and $\mathbf{b}$ a typical targetlike lesion in an axial image.
Fig. 1 Initial colonoscopy in a 47 -year-old man with a cecal adenoma showing a 15 -mm, nonpolypoid lesion (0-lla type) located at the cecal base and an iatrogenic ed polypectomy (inset).

A 47-year-old man underwent a colonoscopic polypectomy to treat a cecal adenoma detected during a screening colonoscopy. The initial colonoscopy showed a $15-\mathrm{mm}$, nonpolypoid lesion (0-Ila type) located at the cecal base ( Fig. 1). After a submucosal injection of $4 \mathrm{~mL}$ of diluted epinephrine solution $(1: 10000)$, the lesion was successfully resected en bloc using a standard snare and electrical current and without complications. Prophylactic hemoclipping was carried out to prevent postprocedural bleeding. Total time taken for the colonoscopy was about 14 minutes.

At 12 hours after the procedure, the patient complained of colicky abdominal pain. Physical examination disclosed localized tenderness in the right lumbar region. Laboratory studies revealed peripheral blood leukocytosis $(13320 / \mu \mathrm{L})$ with neutrophilia (88.9\%), increased levels of C-reactive protein $(1.0 \mathrm{mg} / \mathrm{dL})$, and normal hemoglobin $(14.1 \mathrm{~g} / \mathrm{dL})$. A plain abdominal film excluded pneumoperitoneum. We carried out urgent abdominal computed tomography (CT), which demonstrated a typical targetlike lesion with telescoping of the cecum into the ascending colon ( Fig. 2). Follow-up colonoscopy revealed a markedly congested masslike lesion, which was proven to be the swollen cecal wall as a lead point of intussusception. There was also prominent mucosal discoloration, edema, and exudation in the cecum, suggestive of acute bowel ischemia ( Fig.3) ( Video 1 ). Based on the colonoscopic findings, the patient underwent emergency laparoscopic right hemicolectomy. The pathologic examination confirmed ceco-colic intussusception with ischemic infarction in the cecum without evidence of perforation ( Fig.4).

Intussusception after colonoscopy is an extremely rare phenomenon [1-4]. This unique case highlights the need to consider intussusception as a serious complication of colonoscopic polypectomy. We presume that the precipitating mechanisms included bowel edema secondary to a transmural burn and localized ischemia induced by epinephrine used for sub-

Fig.3 Follow-up colonoscopy showing: a a markedly congested, swollen cecal wall as a lead point; and $\mathbf{b}$ prominent mucosal discoloration, edema, and exudation around the polypectomy site in the cecum.

\section{Video 1}

Follow-up colonoscopy revealing a markedly congested, swollen cecal wall as a lead point of intussusception. Note the mucosal discoloration, edema, and exudation around the polypectomy site, suggestive of acute bowel ischemia. 


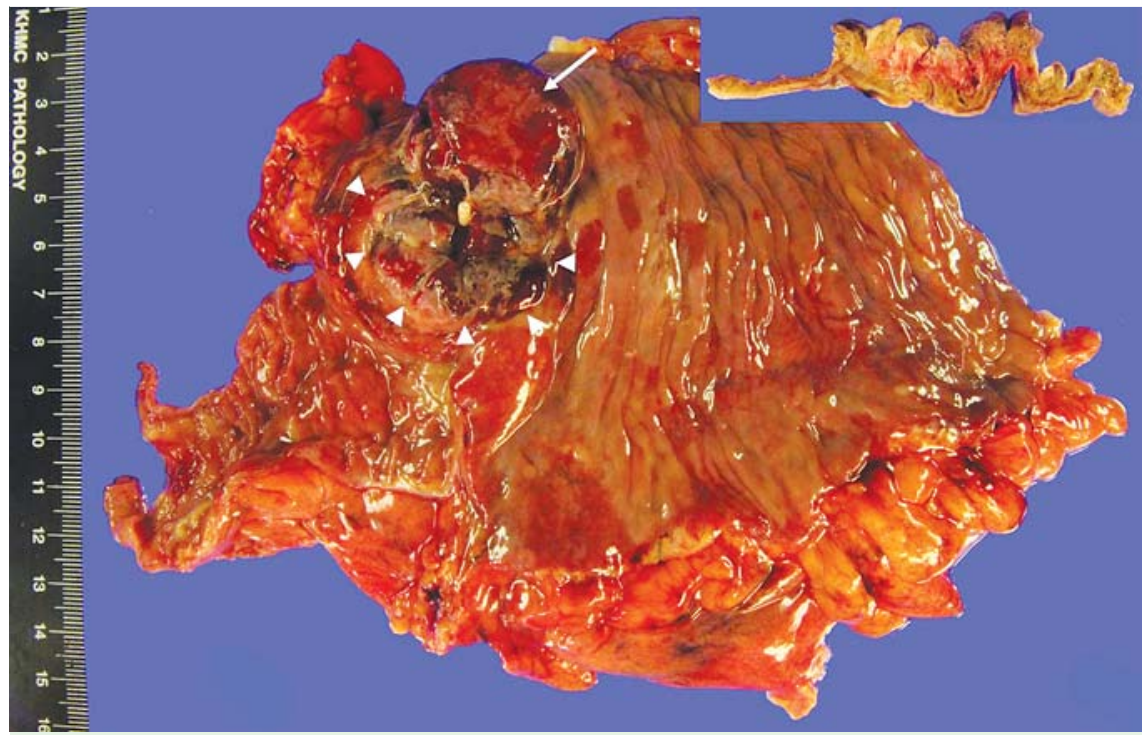

Fig. 4 Gross appearance of the resected specimen and its cut surface (inset) shows a swollen cecal wall as a lead point (arrow) and a well-defined ischemic infarction in the remaining cecum (arrowheads). There was no evidence of perforation at the polypectomy site.

mucosal lifting. Paradoxically, colonoscopy can be useful for the immediate diagnosis of bowel ischemia secondary to intussusception.

Endoscopy_UCTN_Code_CPL_1AJ_2AC

Competing interests: None

\section{References}

1 Azar T, Berger DL. Adult intussusception. Ann Surg 1997; 226: 134-138

2 Wang $N$, Cui XY, Liu Y et al. Adult intussusception: a retrospective review of 41 cases. World J Gastroenterol 2009; 15: 3303 - 3308

3 Ho MM, Park JJ, Prasad LM. Post colonoscopy colonic intussusception reduced via a laparoscopic approach. JSLS 2010; 14: $596-$ 599

4 Nachnani J, Burns E, Margolin D et al. Colocolonic intussusception after colonoscopy. Gastrointest Endosc 2012; 75: 223-225

Bibliography

DOI http://dx.doi.org/

10.1055/s-0032-1326345

Endoscopy 2013; 45: E106-E107

(c) Georg Thieme Verlag KG

Stuttgart · New York

ISSN 0013-726X

\section{Corresponding author}

\section{K. Lee}

Department of Internal Medicine

Kyung Hee University School of Medicine

1 Hoegi-dong

Dongdaemun-gu

Seoul 130-702

Korea

Fax: +82-2-9681848

cklee92@paran.com 